\title{
The interference of rainfall distribution in size of rain water tanks in Bahia
}

\section{Juliana Araújo ${ }^{1}$, Eduardo Cohim²}

${ }^{1}$ Graduanda em Engenharia Civil pela Universidade Estadual de Feira de Santana (UEFS), bolsista Iniciação Científica CNPQ. Feira de Santana-BA

${ }^{2}$ Profes sor titular da Universidade Estadual de Feira de Santana (UEFS). Feira de Santana-BA.

Email: julianafariaz1996@g mail.com, edcohim@gmail.com

Received: March $13^{\text {th }}, 2017$

Accepted: May $14^{\text {th }}, 2017$

Published: June $30^{\text {th }}, 2017$

Copyright $\bigcirc 2016$ by authors and Institute of Technology Galileo of Amazon (ITEGAM). This work is licensed under the Creative Commons Attribution International License (CC BY 4.0).

http://creativecommons.org/lic enses/by/4.0/ (c) (1) (3) Opea Actes:

\begin{abstract}
This work was developed with the aim of contributing to improved design methods of rainwater storage tanks, so we studied the way as an independent variable alone interfere in the design of volume tanks. This variable is the annual distribution of rainfall and, to be carried out this study, we calculated the GCP - degree of concentration of annual rainfall - for the seven (7) studied Bahian cities . Besides calculating this statistic quantity, it was analyzed as it interferes in the volume of the reservoirs was concluded and there is a tendency of larger reservoirs for higher values of GCP.
\end{abstract}

Keywords: Size, cisterns, GCP.

\section{A interferência da distribuição da precipitação no volume dos reservatórios de água pluvial na Bahia}

\section{RESUMO}

Este trabalho foi desenvolvido com o objetivo de contribuir para a melhoria dos métodos de dimensionamento dos reservatórios de armazenamento de água de chuva, de forma que foi estudada a maneira de como uma variável independente, isoladamente, interfere no dimensionamento de volume de reservatórios. Essa variável é a distribuição anual das chuvas e, para que fosse realizado este estudo, foi calculado o GCP - grau de concentração das precipitações anuais - para as 7 (sete) cidades baianas estudadas. Alémde calcular essa grandeza estatís tica, foi analis ado como ela interfere no volume dos reservatórios e concluiu-se há uma tendência de reservatórios maiores para valores mais elevados de GCP.

Palavras Chaves: Dimensionamento, Cisternas, GCP.

\section{INTRODUÇÃO}

Será estudada a variação das chuvas no Estado da Bahia que apresenta um cenário com alta variabilidade na precipitação pluvial, decorrente da união de diferentes sistemas meteorológicos que atuam nessa região, tais como, Zona de Convergência Intertropical (ZCIT), Vórtices Ciclônicos (VCAN), Sistemas Frontais (FPA), Zona de Convergência do Atlântico Sul (ZCAS), ondas de leste, brisas marítimas/terrestres e ventos vale/montanha, bem como de um relevo constituído por planícies, vales, serras e montanhas [1].

É fundamental que se estude a variabilidade da distribuição espaço-temporal das chuvas para que se melhore o aproveitamento da água de chuva, a qual é considerada uma fonte alternativa no que diz respeito ao uso da água para fins potáveis ou não potáveis. Para que is so ocorra, deve-se haver uma melhora nos métodos de dimensionamento de águas pluviais, uma vez que há uma desconfiança da uniformidade da capacidade das cisternas definidas em 16 mil litros no Programa de Formação e Mobilização
Social para a Convivência com o Semiárido - P1MC, no início de 2011, a qual é baseada na demanda de uma família média de cinco pessoas em um período de 280 dias.

Para contribuir com os métodos de dimensionamento, estudará a maneira como uma variável independente, isoladamente, interfere no dimensionamento do volume dos reservatórios. Essa variável é a distribuição anual das chuvas e, para que fosse realizado este estudo, foi calculado o GCP, que indica o grau de concentração das precipitações anuais. Além de calcular essas grandezas, foi analisado como elas interferem no volume de reservatórios.

Comprova-se a necessidade de estudar a interferência da distribuição da precipitação no volume dos reservatórios por conta da ideia de que se dimensionam reservatórios com volumes diferentes, a partir do método de Rippl, para o município de Barreiras e Lençóis que apresentam a mesma média anual de precipitação [2]. Mostra-se assim, que há interferência também da distribuição das precipitações no volume ótimo de reservatório e não apenas das médias pluviométricas. 


\section{MATERIAIS E MÉTODOS}

Para que fosse calculada a distribuição anual das chuvas, foi necessário obter séries diárias, mensais e anuais de precipitação, coletadas empostos dis tribuídos es pacialmente no es tado da Bahia, disponíveis no HidroWeb, da Agência Nacional de Águas (ANA) e no BDRH (Banco de Dados de Recursos Hídricos, do Inema (Instituto do Meio Ambiente e Recursos Hídricos da Bahia). Utilizou-se tanto séries de 15 como de 33 anos no estudo.

Os dados de precipitação apresentaram algumas falhas que precis aram ser corrigidas. Para is so analis aram-se os dados de três postos pluviométricos próximos e fez uso de dois procedimentos. Cada procedimento foi utilizado a depender da variação das precipitações anuais entre esses postos. A partir do preenchimento de falhas, foi feita uma análise de consistência para a homogeneidade dos resultados obtidos [3]

Os 7 (sete) postos estudados apresentam valores de médias de precipitações anuais bem próximas, na faixa entre $723,90 \mathrm{~mm} /$ ano até 757,60 mm/ano. Esses postos serão exibidos a seguir, com suas respectivas precipitações médios anuais (TABELA 1):

TABELA 1: Indica a precipitação média anual dos postos pluviométricos

\begin{tabular}{|c|c|}
\hline $\begin{array}{c}\text { POSTOS } \\
\text { PLUVIOMÉTRICOS }\end{array}$ & $\begin{array}{l}\text { PRECIPITAÇÃO } \\
\text { ANUAL (mm/ano) }\end{array}$ \\
\hline Ibotirama & 723,9 \\
\hline Santa Bárbara & 730,2 \\
\hline Palmas de Monte Alto & 734,5 \\
\hline Saúde & 735,3 \\
\hline Brotas De Macaúbas & 745,1 \\
\hline Serrinha & 746,2 \\
\hline Cândido Sales & 757,6 \\
\hline
\end{tabular}

Fonte: Os autores

Calculou-se o valor médio da precipitação das 7 (sete) cidades e o valor do desvio padrão desses mesmos valores, a fim de que fosse possível calcularo coeficiente de variação, tendo sido encontrado o valor de 1,54\% para o coeficiente de variação, comprovando que os dados de precipitação são homogêneos, ou seja, eles apresentam variação pequena em torno da média $(738,97$ $\mathrm{mm} / \mathrm{ano}$ ).

A escolha por esses postos justifica-se porque o objetivo deste estudo é avaliar a interferência das distribuições das chuvas no tamanho do reservatório. Assim, houve a necessidade de se trabalhar com valores fixos concernentes a outras variáveis, quais sejam, área de telhado, demanda, precipitação média anual e confiabilidade ou nível de risco aceitável (percentual de dias em que a demanda é plenamente atendida em um dado período de análise) [4].

Dessa forma, foi importante buscar valores próximos de precipitações médias anuais para que fosse possível manter essa variável fixa no estudo. Além dela, foram fixadas outras variáveis, como: área de telhado como $80 \mathrm{~m}^{2}$, confiabilidade como $90 \%$. Realizou-se o estudo para três valores demanda (50 1/dia, 70 1/dia, e 90 Ldia).

A fim de que fossem realizadas as simulações de volume de reservatório de águas pluviais, utilizou-se o Software Netuno [5].

A escolha pelo uso do GCP ocorreu porque ela é capaz de identificar a heterogeneidade temporal na precipitação. Essa grandeza já foi utilizada por Zhang e Qian (2003), quando analisaram as características da distribuição espacial e temporal e variação de precipitação em uma região da China. Ela também já foi utilizada por Li et al. (2011), em que se estudou a variabilidade espacial e temporal de precipitação em Xinjiang, na China, em que foi possível constatar que a precipitação no norte da região foi mais dispersa dentro de um ano do que no sul.

Para realizar o cálculo do GCP (grau de concentração de precipitação) para os 7 (sete) postos pluviométricos, partiu-se do pressuposto de que as precipitações mensais são grandezas vetoriais, orientadas em um círculo trigonométrico, em que a direção destes vetores é determinada em função do mês de ocorrência, em que cada mês as sume um valor de $30^{\circ}$.

Assim, o mês de janeiro fica como ângulo de $0^{\circ}$ e dezembro como ângulo de $330^{\circ}$, sendo que a abrangência de cada mês é de (+/-) $15^{\circ}$. O valor do GCP varia de 0 a 1 , sendo que os valores próximos a 0 representam chuvas mais distribuídas ao longo do ano, enquanto que valores próximos a 1 indica a concentração das chuvas em um curto período. Utilizam-se as seguintes equações (Equações 1, 2, 3,4) para calcular o GCP [6].

$$
\begin{gathered}
\boldsymbol{R}_{i}=\sum \boldsymbol{r}_{i j} \\
\boldsymbol{R}_{x i}=\sum \boldsymbol{r}_{i j} \cdot \sin \theta_{j} \\
\boldsymbol{R}_{y i}=\sum \boldsymbol{r}_{i j} \cdot \cos \theta_{j} \\
\mathbf{G C P}_{\mathrm{ij}}=\frac{\sqrt{\mathbf{R}^{2}{ }_{\mathrm{xi}}+\mathbf{R}^{2} \mathbf{y i}}}{\mathbf{R}_{\mathbf{i}}}
\end{gathered}
$$

Em que, $i$ é o ano da série histórica, $j$ representa o mês, $r_{i j}$ demonstra a precipitação de um mês de um determinado ano e o $\theta_{j}$ é o ângulo representa ao mês que se está estudando.

No decorrer da realização do estudo, observou-se que para determinar o GCP para um dado posto selecionado é necessário calcular as médias de precipitações para cada mês da série his tórica e, em seguida calcular o GCP com essas médias. Em seguida, construíram-se gráficos, os quais analis avam a GCP versus volume do reservatório para cada demanda. As conclusões obtidas com esse estudo serão discutidas mais adiante.

\section{RESULTADOS E DISCUSSÃO}

Foram encontrados os valores de GCP e esses resultados estão presentes na Tabela 2, a seguir:

Tabela 2: Relação dos postos pluviométricos comseus respectivos valores de GCP.

\begin{tabular}{|c|c|}
\hline POSTOS & GCP \\
PLUVIOMÉTRICOS & MÉDIA \\
\hline Serrinha & 0,122 \\
\hline Santa Bárbara & 0,132 \\
\hline Saúde & 0,298 \\
\hline Cândido Sales & 0,472 \\
\hline Brotas De Macaúbas & 0,595 \\
\hline Ibotirama & 0,607 \\
\hline Palmas de Monte Alto & 0,642 \\
\hline
\end{tabular}

Fonte: Autores, (2016). 
Da mesma maneira que se calcularam grandezas estatísticas para os valores de precipitação, foi calculado a média dos valores de GCP, o desvio padrão e o coeficiente de variação. Encontrou-se o valor médio de GCP como 0,41 e o coeficiente de variação como $54,91 \%$. Percebe-se que o valor do coeficiente de variação do GCP é alto e esse fato era uma exigência do trabalho, afinal busca-se estudar a interferência da variação do Grau De Concentração da Precipitação na determinação do volume dos reservatórios de água pluvial, assim fazem-se necessários valores bem distintos de GCP para que o estudo seja mais abrangente.

Após essa etapa, foram feitos e analisados 3 (três) gráficos que relacionam os GCP calculados com o volume do reservatório encontrado no Netuno. O gráfico da figura 1 analisa GCP versus Volume para a demanda de $50 \mathrm{l} / \mathrm{dia}$. O gráfico da figura 2 faz a mesma análise para a demanda de 70 1/dia e o gráfico 3 para a demanda de 90 1/dia. Esses gráficos são exibidos a seguir:

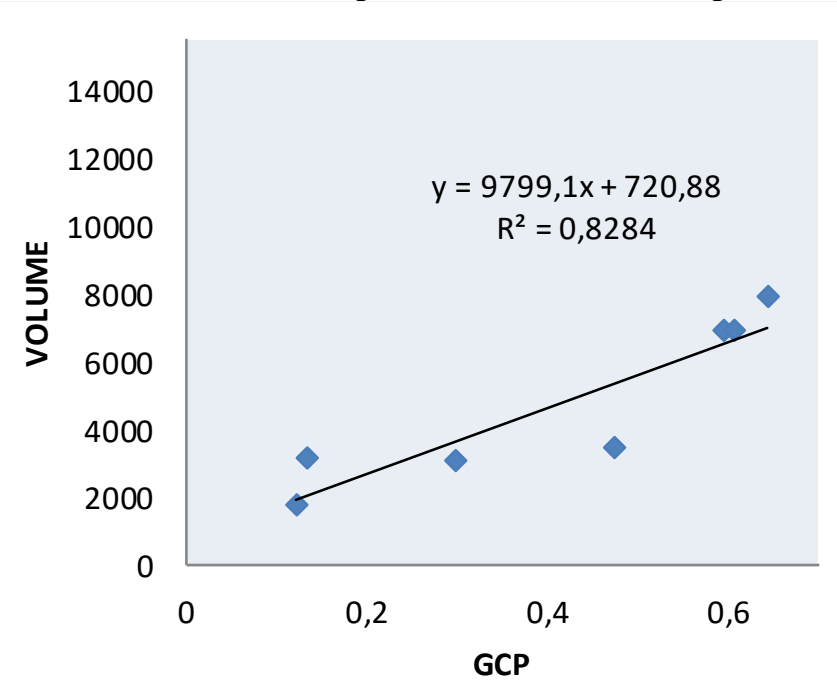

Figura 1: Gráfico- Relação entre os GCP calculados e o volume $\left(\mathrm{m}^{3}\right)$ para os 7 (sete) postos pluviométricos para demanda de 50 Ldia.

Fonte: Autores, (2016).

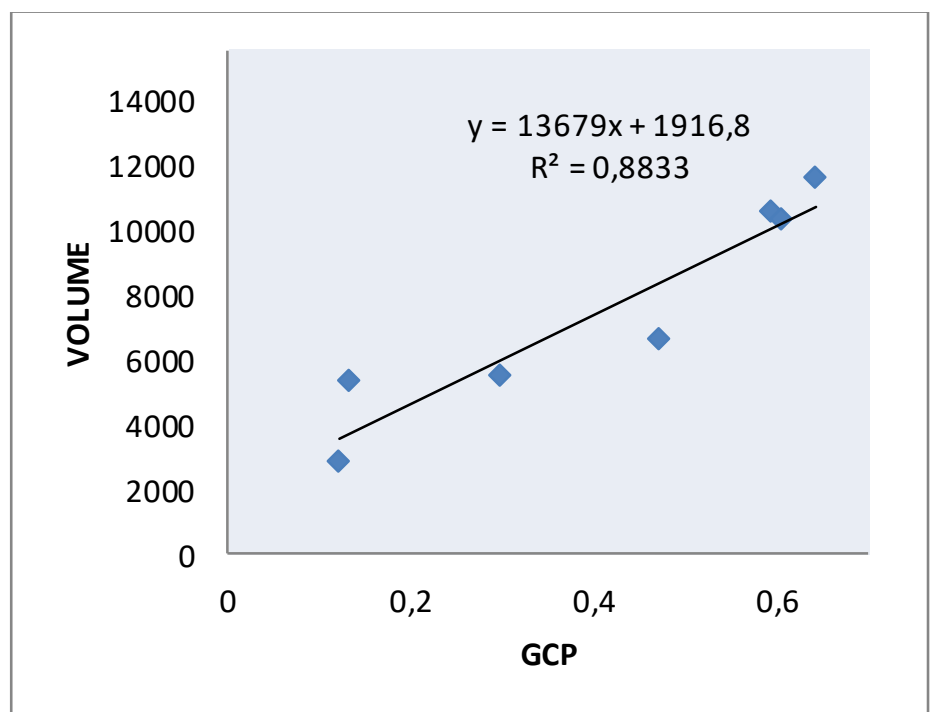

Figura 2: Gráfico - Relação entre os GCP calculados e o volume $\left(\mathrm{m}^{3}\right)$ para os 7 (sete) postos pluviométricos para demanda de 70 Ldia.

Fonte: Autores, (2016).

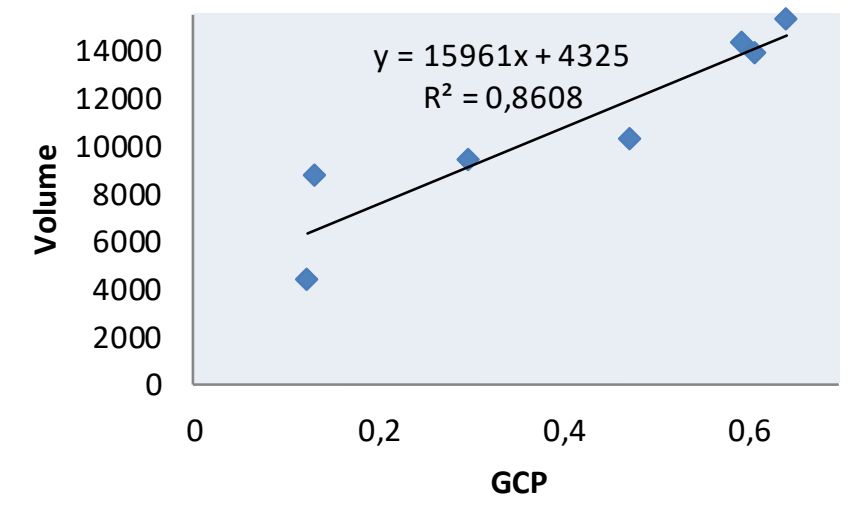

Figura 3: Gráfico - Relação entre os GCP calculados e o volume $\left(\mathrm{m}^{3}\right)$ para os 7 (sete) postos pluviométricos para demanda de 90 Ldia.

Fonte: Autores, (2016).

Percebe-se que os 3 gráficos apresentam linhas de tendências lineares com valores de coeficiente de determinação $\left(\mathrm{R}^{2}\right)$ bem elevados (maiores do que 0,8 ), mostrando que, de fato, existe uma relação entre o GCP com o volume de reservatório.

Pode-se concluir com a interpretação dos dados apresentados nos gráficos que correlacionam GCP com o volume que, para uma dada confiabilidade e com os valores de demanda fixos, quanto maior o valor do GCP, maior será o volume dos reservatórios de água pluvial encontrado a partir do Software Netuno.

Esse comportamento pode ser exemplificado ao analisar o gráfico GCP versus Volume para uma demanda de 90 1/dia, em que o valor encontrado no Software Netuno para o GCP de 0,642 corresponde a $15300 \mathrm{~m}^{3}$, enquanto o encontrado para o GCP de 0,122 corresponde a $4350 \mathrm{~m}^{3}$. Esse comportamento é o esperado, afinal quanto maior o valor do GCP maior concentração das precipitações anuais. Dessa forma, é necessário armazenar a água em reservatórios para que se possa usar esse bem em períodos de estiagem. Isso acontece, principalmente, em locais que não dispõem de outra fonte de abastecimento de água.

Além disso, percebeu-se que os volumes encontrados para o GCP de 0,595 e de 0,607 são bem próximos e em algumas situações existiu um pequeno decréscimo de valor do volume para o GCP de 0,595 (Brotas de Macaúbas) para o volume para o GCP de 0,607 (Ibotirama). Esse comportamento não é esperado, mas pode ser explicado devido à pequena variação nos valores de precipitação média anual, em que o posto de Brotas de Macaúbas apresenta uma precipitação média anual de $745,1 \mathrm{~mm} /$ ano e o posto de Ibotirama de 723,9 mm/ano.

\section{CONCLUSÕES}

Considera-se, dessa forma, que o estado da Bahia apresenta uma grande variabilidade da distribuição espaçotemporal das chuvas e que o estudo dessa variável é fundamental para o melhor aproveitamento da água pluvial, principalmente, para melhor dimensionar os reservatórios de águas das chuvas. Assim, é fundamental estudar como a dis tribuição anual das chuvas interfere no dimensionamento dos reservatórios, a partir de grandezas estatísticas o Grau de Concentração da Precipitação (GCP). A partir deste estudo, percebe-se que quanto maior o GCP, em uma dada confiabilidade e numa dada demanda, maior será o valor do volume encontrado no programa Netuno. Esses resultados 
apontam para a necessidade de se armazenar a água no período chuvoso, para que possa ser utilizada em períodos de estiagem, culminando na necessidade de maiores reservatórios.

\section{REFERÊNCIAS}

[1] Barbosa, D.V.N. (1998). Os Impactos da Seca de 1993 no Semiárido Baiano: Caso de Irecê. Salvador: SEI, 2000. In:

[2] Cohim, Eduardo; Garcia, Ana; Kiperstok, Asher (2008). Captação e utilização de água pluvial em residências para população de baixa renda em áreas urbanas: Estudo de caso.

[3] Simões, Yagho; Cohim, Eduardo; Araujo, Heráclio (2014). Análise de tendências do comportamento das chuvas no estado da bahia. Apresentação no 9 Simpósio brasileiro de captação manejo água chuva. Feira de Santana- BA.

[4] Cohim, Eduardo; Orrico, Sílvio. (2015). A confiabilidade do volume das cisternas da zona rural para reservar água de chuva. Revista eletrônica de gestão e tecnologias ambientais. GESTA, v. 3, n. 2 -, p. 91-99.

[5] Ghisi, E.; Cordova, M. M. Netuno: Manual Do Usuário. Disponível

em: <http://www.labeee.ufsc.br/sites/default/files/Manual-Netuno4_Junho2014.pdf> Acesso em: 23.dez.2015.

[6] Li, X.; Jiang, F.; Li, L. Wang, G. (2011). Spatial and temporal variability of precipitation concentration index, concentration degree and concentration period in Xinjiang, China. Int. J. Climatol. 31: 1679-1693.

[7] Braga, C.C; Melo, M.L.D; Melo, E.C.S. Análise de Agrupamento Aplicada a Distribuição da Precipitação no Estado da Bahia. $10^{\circ}$ Congresso Brasileiro de Meteorologia. Brasília-DF. Anais... Sociedade Brasileira de Meteorologia. p.1857-62, 1998.

[8] Braga, C. C.; Melo, M. L. D.; Melo, E. C. S. (1998). Análise de agrupamento aplicada a distribuição da precipitação no Estado da Bahia. In: Congresso Brasileiro de Meteorologia, 10, 1998, Brasília. Anais... Brasília: Sociedade Brasileira de Meteorologia, 1998. CD-Room.

[9] Zhang, L. J.; Qian, Y. F. (2003). A study on the feature of precipitation concentration and its relation to flood-producing in the Yangtze River Valley of China. Chinese journal of geophysics. 47: 709-718. 